\title{
Young, American Indian or Alaskan Native, and born in the USA: At excess risk of starting extra-medical prescription pain reliever use?
}

\author{
Maria A Parker ${ }^{\text {Corresp., }}{ }^{1,2}$, Catalina Lopez-Quintero ${ }^{1,3}$, James C Anthony ${ }^{1}$ \\ 1 Department of Epidemiology \& Biostatistics, Michigan State University, East Lansing, Michigan, United States \\ 3 Department of Epidemiology, University of Florida, Gainesville, FL, United States \\ Corresponding Author: Maria A Parker \\ Email address: maria.parker@uvm.edu
}

Background. Prescription pain reliever (PPR) overdoses differentially affect 'American Indian/Alaskan Natives' in the United States (US). Here, studying onset of extra-medical PPR use in 12-24 year-olds, we examine subgroup variations in rates of starting to use prescription pain relievers extra-medically (i.e., to get 'high' or for other reasons outside boundaries of prescriber's intent). Risk differences (RD) are estimated for US-born versus non-US-born young people, stratified by American Indian/Alaskan Natives versus other ethnic self-identities.

Methods. Between 2002-2009, nationally representative cross-sectional samples of 12-24-year-old noninstitutionalized civilians completed interviews for the US National Surveys of Drug Use and Health. Analysis-weighted annual incidence estimates, RD, and confidence intervals $(\mathrm{Cl})$ are from the Restricteduse Data Analysis System, an online software tool for US National Surveys of Drug Use and Health.

Results. Each year, an estimated $2.5 \%$ of 12 -24-year-olds in the US start using PPR extra-medically (95\% $\mathrm{Cl}=2.1 \%, 3.0 \%)$. Estimates for the US-born (3.8\%; 95\% Cl=3.7\%, 3.9\%) are larger (non-US-born: $1.8 \% ; 95 \% \mathrm{Cl}=1.5 \%, 2.0 \% ; \mathrm{RD}=2.0 ; \mathrm{p}<0.05)$. US-born American Indian/Alaskan Natives youths have the largest incidence rate (4.8\%). Robust RD for US-born can be seen for 'non-Hispanic White' subgroups, and for others (e.g., 'Cuban', 'Dominican').

Discussion. Each year, one in 20 of US-born American Indian/Alaskan Natives starts using PPR extramedically. Overdose prevention is important, but is no substitute for primary prevention initiatives for all young people. The observed epidemiological patterns can guide targeted prevention initiatives for the identified higher risk subgroups in complement with more universal prevention efforts intended to reduce incidence of first extra-medical PPR use, a crucial rate-limiting step on the path toward more serious drug involvement (i.e., progressing past initial use). 
1

2

3

4

5

6 Young, American Indian or Alaskan Native, and Born in the USA: At Excess Risk

8

9

10

11

12

13

14

15 Correspondence: Dr. Maria A. Parker, Vermont Center on Behavior \& Health, University of

16 Vermont, Burlington, VT, United States, Tel.: +1 8026560206 Fax: +1 802 656-5793. Email

17 address: maria.parker@uvm.edu
PLEASE DO NOT QUOTE OR CITE UNTIL 'IN PRESS'

PeerJ

Version 27 August 2018
Word Count: 1,964

${ }^{1}$ Michigan State University, Department of Epidemiology \& Biostatistics, East Lansing, MI, United States

${ }^{2}$ University of Vermont, Department of Psychiatry, Burlington, VT, United States

${ }^{3}$ University of Florida, Department of Epidemiology, Gainesville, FL, United States 
19 ABSTRACT (word count: 267/500)

21 Background. Prescription pain reliever (PPR) overdoses differentially affect 'American

22 Indian/Alaskan Natives' in the United States (US). Here, studying onset of extra-medical PPR

23 use in 12-24 year-olds, we examine subgroup variations in rates of starting to use prescription

24 pain relievers extra-medically (i.e., to get 'high' or for other reasons outside boundaries of

25 prescriber's intent). Risk differences (RD) are estimated for US-born versus non-US-born young

26 people, stratified by American Indian/Alaskan Natives versus other ethnic self-identities.

27 Methods. Between 2002-2009, nationally representative cross-sectional samples of 12-24-year-

28 old non-institutionalized civilians completed interviews for the US National Surveys of Drug

29 Use and Health. Analysis-weighted annual incidence estimates, RD, and confidence intervals

30 (CI) are from the Restricted-use Data Analysis System, an online software tool for US National

31 Surveys of Drug Use and Health.

32 Results. Each year, an estimated $2.5 \%$ of $12-24$-year-olds in the US start using PPR extra-

33 medically $(95 \% \mathrm{CI}=2.1 \%, 3.0 \%)$. Estimates for the US-born $(3.8 \% ; 95 \% \mathrm{CI}=3.7 \%, 3.9 \%)$ are

34 larger (non-US-born: $1.8 \% ; 95 \% \mathrm{CI}=1.5 \%, 2.0 \% ; \mathrm{RD}=2.0 ; \mathrm{p}<0.05$ ). US-born American

35 Indian/Alaskan Natives youths have the largest incidence rate (4.8\%). Robust RD for US-born

36 can be seen for 'non-Hispanic White' subgroups, and for others (e.g., 'Cuban', 'Dominican').

37 Discussion. Each year, one in 20 of US-born American Indian/Alaskan Natives starts using PPR

38 extra-medically. Overdose prevention is important, but is no substitute for primary prevention

39 initiatives for all young people. The observed epidemiological patterns can guide targeted

40 prevention initiatives for the identified higher risk subgroups in complement with more universal

41 prevention efforts intended to reduce incidence of first extra-medical PPR use, a crucial rate- 
42 limiting step on the path toward more serious drug involvement (i.e., progressing past initial 43 use).

44 Introduction

Current indicators tracking the prescription pain reliever (PPR) epidemic in the United States (US) reflect several epidemiological processes, including increased clinician prescribing 47 of opioid compounds (King et al., 2014). Concurrently, upward-shifting population incidence rates for starting to use PPR in an 'extra-medical' fashion have been seen - e.g., for feeling states such as to get high and otherwise outside boundaries intended by a prescribing clinician (Parker 50 \& Anthony, 2015).

Newer US estimates suggest recent stabilization of a previously documented upwardturning PPR epidemic curve (United States, 2016). Nevertheless, PPR dependence cases continue to require treatment resources. To illustrate, an estimated $6-9 \%$ of youths starting to use

54 PPR extra-medically become newly incident opioid dependence cases within 12 months after first extra-medical use (Parker \& Anthony, 2015). Fatal and non-fatal overdoses motivate concern and prompt public health initiatives to reduce extra-medical PPR use (Compton \& Volkow, 2006). Indian/Alaskan Native heritage ('Native Americans') generally have been neglected (Kochanek et al., 2016; Mack, 2017). While Native Americans have the highest drug overdose death rates,

61 these rates have not yet abated and continue to increase. Overdose death rates sometimes can be 62 traced to more rapid acceleration of extra-medical drug experiences once first use has occurred.

63 However, the underlying explanation might be larger first-inception incidence rates for starting 64 extra-medical PPR use - i.e., larger for Native Americans (Stanley et al., 2014). 
Among those who start using PPR extra-medically, mean and median age values are well

66

67

68

69 under 25 years, and initiation rates for 12-17-year-olds do not differ appreciably from those in young adulthood (e.g., 18-24-year-olds; Parker \& Anthony, 2015; United States, 2016). Hence, it is important to examine estimates focused on 12-24-year-olds.

Quite plausibly, US-born young people in this age range might be generally more likely to start using PPR when compared with their non-US-born immigrant peers, and rates of starting extra-medical PPR use might be greater for US-born Native Americans versus those born outside the US (United States, 2013). For this reason, we organized the analysis approach to compare US versus non-US born populations, with a focus on US-born vs. non-US born American Indian/Alaskan Native (e.g., belonging to Central or South American Indian groups). By focusing on US young people's PPR risk experiences in 2002-2009, we leave an opportunity for evaluation of reproducibility when more recent data become available for 2010 through 2017.

Our hope is that the results, once replicated in newer datasets from 2010-2017, will help guide plans for ending the US opioid epidemic (Kolodny \& Frieden, 2017).

\section{Materials \& Methods}

\section{Study Population, Participants, \& Data Collection}

The study population consists of non-institutionalized US community residents age 12-

24-years-old, as sampled, recruited, and assessed using Institutional Review Board approved protocols for National Surveys on Drug Use and Health (NSDUH). These surveys involved drawing new nationally representative samples each year, 2002-2009, generally with $>70 \%$ participation levels (United States, 2013). Publications and online reports provide detailed descriptions of NSDUH methods and the no longer available Restricted-use Data Analysis System (RDAS) datafiles that enabled stratification by US birthplace (e.g., 
88 http://www.samhsa.gov/data/population-data-nsduh/reports; (United States, 2013;

89 Vsevolozhskaya \& Anthony, 2014).

90 Unlike other datasets, the formerly available RDAS datafiles for 2002-2009 covered

91 more than 200,000 12-24-year-olds with detailed variables on ethnic self-identification (ESI;

92 beyond the limited race-ethnicity subgroups of US federal research:

93 https:/grants.nih.gov/grants/funding/women_min/inclusion_ims.htm) and US-born status.

94 Participation involved completion of audio computer self-interviews (ACASI) with standardized

95 multi-item modules.

96 Measures

97 The ACASI items on extra-medical PPR use identify never-users, past-onset users, and

98 'past-year initiates,' as described in detail previously (Parker \& Anthony, 2015). ACASI ESI

99 items ask "Which of these groups describes you?" with detailed responses: Mexican/Mexican

100 American/Chicano, Puerto Rican, Central or South American, Cuban/Cuban American,

101 Dominican (i.e., from the Dominican Republic), Chinese, Filipino, Indian (Asian), Vietnamese,

102 Korean, Japanese, Other Asian (non-specified), non-Hispanic White, non-Hispanic

103 Black/African American, American Indian or Alaskan Native (herein abbreviated as 'Native

104 American'), Native Hawaiian, Other Pacific Islander (excluding Native Hawaiian), Other (non-

105 specified).

106 Analysis

107 Analysis-weighted incidence rates reported here are from RDAS variables designating a

108 numerator ['past-year initiates' or newly incident users with extra-medical PPR onset within 12

109 months before assessment], and a denominator of all 'at risk' of doing so. Respondents with no

110 use of extra-medical PPR (in the lifetime) as well as the newly incident users were counted in the 
111 denominators of this study's incidence rates as members of the 'at-risk' population. The

112 denominator excluded past-onset users because they no longer are candidates for becoming

113 newly incident users.

114 In this fashion, annual incidence rates were formed for 18 ESI subgroups, and risk

115 differences (RD) were calculated to contrast US-born and non-US-born immigrant experiences.

116 RD estimates are formed by subtracting each subgroup-specific incidence rate for non-US-born

117 immigrants from the corresponding US-born estimate. RDAS provided analysis weights

118 appropriate for pooled analyses of 2002-2009 data bringing age and sex into balance with US

119 Census distributions via Taylor series linearization standard errors (United States, 2013;

120 Vsevolozhskaya \& Anthony, 2014). We derived 95\% confidence intervals (CI) and evaluated

121 null hypotheses at $\mathrm{RD}=0$ with alpha at 0.05 .

122 Results

123 Table 1 depicts unweighted subgroup sample sizes, detailed annual incidence estimates

124 for ESI and birthplace subgroups, and RD. The overall incidence rate estimate for US 12-24-

125 year-olds is $2.5 \%(95 \% \mathrm{CI}=2.1 \%, 3.0 \%)$ - i.e., an estimated two to three newly incident users per

126100 12-24-year-olds (unweighted denominator $\mathrm{n}=173,961$ ). Corresponding estimates for non-

127 US-born immigrants versus the US-born were $1.8 \%(95 \% \mathrm{CI}=1.5,2.0)$ versus $3.8 \%(95 \%$

$128 \mathrm{CI}=3.7,3.9)$, respectively, with $\mathrm{RD}=2.0(\mathrm{p}<0.05$; Table 1). Evaluated for all 36 subgroups under

129 study, the largest observed estimate is 4.5\%, seen for Native American 12-24-year-olds

$130(n=2,031)$, with excess risk concentrated among the US-born $(4.8 \% ; \mathrm{p}<0.05$; Table 1$)$.

131 Table 1 also shows several ESI subgroups with distinctively large incidence rates,

132 including non-Hispanic Whites and the Hispanic subgroups with ESI as 'Cuban' or as

133 'Dominican.' Distinctively lower incidence rates are seen for 'Chinese' as well as 'Other Asians' 
134 (i.e., 'not otherwise specified as Asian'). The estimated RD is $2.0 \%$ for all 12-24-year-olds

135 ( $\mathrm{SE}=0.1$ ), versus 3.0\% for those of Native American heritage ( $\mathrm{SE}=0.6$; Table 1, RD Estimates).

136 Figure 1 depicts these incidence estimates and RD in two dimensions. The $\mathrm{x}$-axis

137 arranges incidence estimates for US-born of each subgroup from smallest (Other Asian, 1.1\%) to

138 largest (Native American, 4.8\%). The y-axis presents estimated RD and 95\% CI, showing the

139 just-mentioned excess risk for the US-born among 12-24-year-olds generally $(\mathrm{RD}=2.0 \%)$. Non-

140 null $\mathrm{RD}$ are seen for non-Hispanic Whites $(\mathrm{RD}=2.4 \%)$, and corresponding ESI subgroups of

141 Native Americans $(\mathrm{RD}=3.0 \%)$; Cubans $(\mathrm{RD}=3.2 \%)$, Dominicans $(\mathrm{RD}=3.0 \%)$, and Mexicans

$142(\mathrm{RD}=1.5 \%)$, for which each $95 \% \mathrm{CI}$ fails to touch the $\mathrm{x}$-axis at $\mathrm{y}=0$ (Figure 1$)$. The

143 preponderance of Western Hemisphere subgroups is noteworthy in this list, as is under-

144 representation of Asians. For other subgroups, RD estimates are smaller and statistically

145 imprecise, but larger rates generally are seen for US-born. The Chinese young people provide an

146 unexplained exception to the general rule, but we note that the $95 \% \mathrm{CI}$ for this estimate crosses

147 the $\mathrm{x}$-axis at $\mathrm{y}=0$, and denotes an approximate $\mathrm{p}>0.05$.

148 Discussion

149 It can be useful to conceptualize drug involvement across a spectrum of experiences that

150 range from an initial chance to try a drug and onward toward more serious experiences such as

151 addictive states or overdose. In this work, we focus on early stages of PPR involvement among

152 young people, namely, the first occasion of extra-medical PPR use. These novel epidemiological

153 estimates describe but cannot explain how it happens that this country's US-born young people

154 of Native American heritage have such large incidence rates, versus lower non-US-born rates, as

155 first documented here. However, the observed larger overdose rates for this US subgroup might 
156 be traced, at least in part, to a failure of current programs to prevent extra-medical PPR use

157 (before it starts) among young people of Native American heritage.

158 Paired with the high rates of clinician prescribing in the US (Manchikanti et al., 2012), it

159 may be that opioid public health initiatives also are not reaching many of the Tribal

160 communities. Targeted prevention initiatives for Native Americans such as school screenings or

161 community education efforts complemented with more universal prevention effects could reduce

162 the incidence of first extra-medical PPR use (e.g., The Truth about Opioids:

163 opioids.thetruth.com).

164 These annual incidence rate estimates offer a reminder that preventive medicine must

165 complement opioid dependence treatment and overdose services (Kolodny \& Frieden, 2017).

166 Epidemiological estimates of this type also can be useful as differentially affected population

167 subgroups may have been overlooked.

168 Before detailed discussion of these results, several of the more important study

169 limitations merit attention, including the cross-sectional NSDUH design, the self-reported nature

170 of the data, and the somewhat historical nature of the 2002-2009 datasets. However, if we can

171 assume that ESI and US-born status qualify as variables that are relatively time-invariant, and do

172 not change after onset of extra-medical PPR use, then the research design does not create

173 inferential difficulties that epidemiologists face when time-varying and drug-responsive

174 characteristics are investigated at cross-section. We do note, however, some smaller sample sizes

175 where the RD could not be estimated (i.e., Japanese and Native Hawaiian). As for the historical

176 nature of these data, we had anticipated release of NSDUH data from 2010 onward, but the US

177 federal agencies have delayed release of these more recent datasets. Datasets from surveys 
178 completed during 2010-2017 should make it possible to replicate this study's estimates, or to

179 disconfirm them.

180 This epidemiological study represents a departure from prior studies focused on

181 prevalence of PPR use - that is, being a user. In contrast, our study concerns incidence or

182 becoming an extra-medical PPR user for the first time. During 2002-2009, there is clear evidence

183 that US-born subgroups generally experienced excess risk as compared with non-US-born

184 immigrants. ESI subgroups from the Western Hemisphere serve best to illustrate this variation in

185 risk experience. In ESI subgroups from Asian countries, smaller incidence rates and a smaller

186 US-born excess generally is seen.

187 We are hopeful that these initial clues about uneven distribution of PPR incidence rates

188 will set up new lines of study, including investigation of male-female differences within

189 implicated subgroups as well as potential effects of time since immigration to the US. Future

190 research should explore whether the effects of time since immigration might be modulated by

191 age at immigration or by within-household characteristics such as language spoken at home.

192 We have left the opportunity for evaluation of reproducibility with newer NSDUH data

193 from 2010-2017, in extension of what we have provided as estimates from mid-epidemic 2002-

1942009 surveillance analyses. For this reason, we decided to seek publication of our initial 2002-

1952009 estimates, and we are hopeful about confirmation with data from more recent years.

196 Nonetheless, we believe it will take a combined qualitative and mixed methods research

197 approach to illuminate generating mechanisms for the observed excess PPR rates, which might

198 include historical/social trauma/norms of US-born Native American youth; Stanley et al., 2014;

199 Stanley, Swaim \& Dieterich, 2017). 
200 Much remains to be learned on the topic of drug involvement in relation to immigrant

201 health. Here, we provide preliminary evidence of what is likely to become recognized as a more

202 important source of variation in the health of subpopulations defined by immigrant status -

203 namely, the degree to which immigrants retain the drug-taking practices of their countries of

204 origin versus adoption of the drug-taking norms in their new neighborhoods.

\section{Conclusion}

206 Looking into the earliest stages of drug involvement, here with emphasis on first extra-

207 medical drug use, we see that young people born in the US are at greater risk of starting to use

208 PPR extra-medically, as compared with non-US-born immigrant peers. Native Americans, an

209 important but often neglected subgroup of the US population, apparently have the largest annual

210 incidence rate for onset of extra-medical use of PPRs. With focus upon first-time drug users, this

211 work's epidemiological estimates are especially pertinent for public health workers and leaders

212 who are responsible for primary prevention of use. 


\section{References}

214 Compton WM., Volkow ND. 2006. Major increases in opioid analgesic abuse in the United

215 States: concerns and strategies. Drug and alcohol dependence 81:103-107. DOI:

$216 \quad$ 10.1016/j.drugalcdep.2005.05.009.

217 King NB., Fraser V., Boikos C., Richardson R., Harper S. 2014. Determinants of increased opioid-related mortality in the United States and Canada, 1990-2013: a systematic review. American Journal of Public Health 104:e32-42. DOI:

221

222

Kochanek KD., Murphy SL., Xu J., Tejada-Vera B. 2016. Deaths: Final data for 2014. National

223 224 225 226

227

228

229

230

231

232

233

234

235 vital statistics reports. Hyattsville, MD: National Center for Health Statistics.

Kolodny A., Frieden TR. 2017. Ten steps the federal government should take now to reverse the opioid addiction epidemic. JAMA 318:1537-1538. DOI: 10.1001/jama.2017.14567.

Mack KA. 2017. Illicit Drug Use, Illicit Drug Use Disorders, and Drug Overdose Deaths in Metropolitan and Nonmetropolitan Areas — United States. MMWR. Surveillance Summaries 66. DOI: 10.15585/mmwr.ss6619a1.

Manchikanti L., Helm S., Fellows B., Janata JW., Pampati V., Grider JS., Boswell MV. 2012. Opioid epidemic in the United States. Pain Physician 15:ES9-38.

Parker MA., Anthony JC. 2015. Epidemiological evidence on extra-medical opioid prescription use: Rapid transitions from newly incident use to dependence among 12-21 year olds in the United States using meta-analysis, 2002-2013. PeerJ 3:e1340.

Stanley LR., Harness SD., Swaim RC., Beauvais F. 2014. Rates of Substance Use of American Indian Students in 8th, 10th, and 12th Grades Living on or Near Reservations: Update, 2009-2012. Public Health Reports 129:156-163. 
236 Stanley LR., Swaim RC., Dieterich SE. 2017. The Role of Norms in Marijuana Use Among

237 American Indian Adolescents. Prevention Science 18:406-415. DOI: 10.1007/s11121-

238 017-0768-2.

239 United States. 2013. National Survey on Drug Use and Health: 2-Year R-DAS (2002 to 2003, 2004 to 2005,2006 to 2007 , and 2008 to 2009 ).

241 United States. 2016. Results from the 2015 National Survey on Drug Use and Health: Detailed Health Services Administration.

244 Vsevolozhskaya OA., Anthony JC. 2014. Confidence interval estimation in R-DAS. Drug and Alcohol Dependence 143:95-104. DOI: 10.1016/j.drugalcdep.2014.07.017. 


\section{Table $\mathbf{1}$ (on next page)}

Estimated annual incidence of extra-medical prescription pain reliever use for 12-24year-olds in the United States (US), stratified by ethnic self-identification subgroups and birthplace. $^{\text {a }}$

Note:

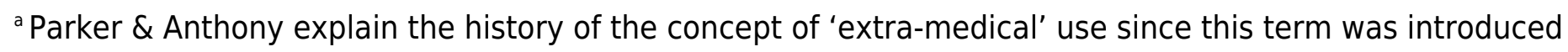
(2015).

${ }^{\mathrm{b}}$ Bolding denotes statistical significance at the alpha $=0.05$ level.

c Unweighted RDAS subgroup size approximations with overall sum at risk for starting extra-medical prescription pain reliever use. RDAS output yields weighted estimates but does not disclose unweighted cell counts, which we have derived using an approximation method (Vsevolozhskaya \& Anthony, 2014).

${ }^{d}$ Not all incidence rates for Japanese and Native Hawaiian by birthplace were estimable due to too few newly incident users. 


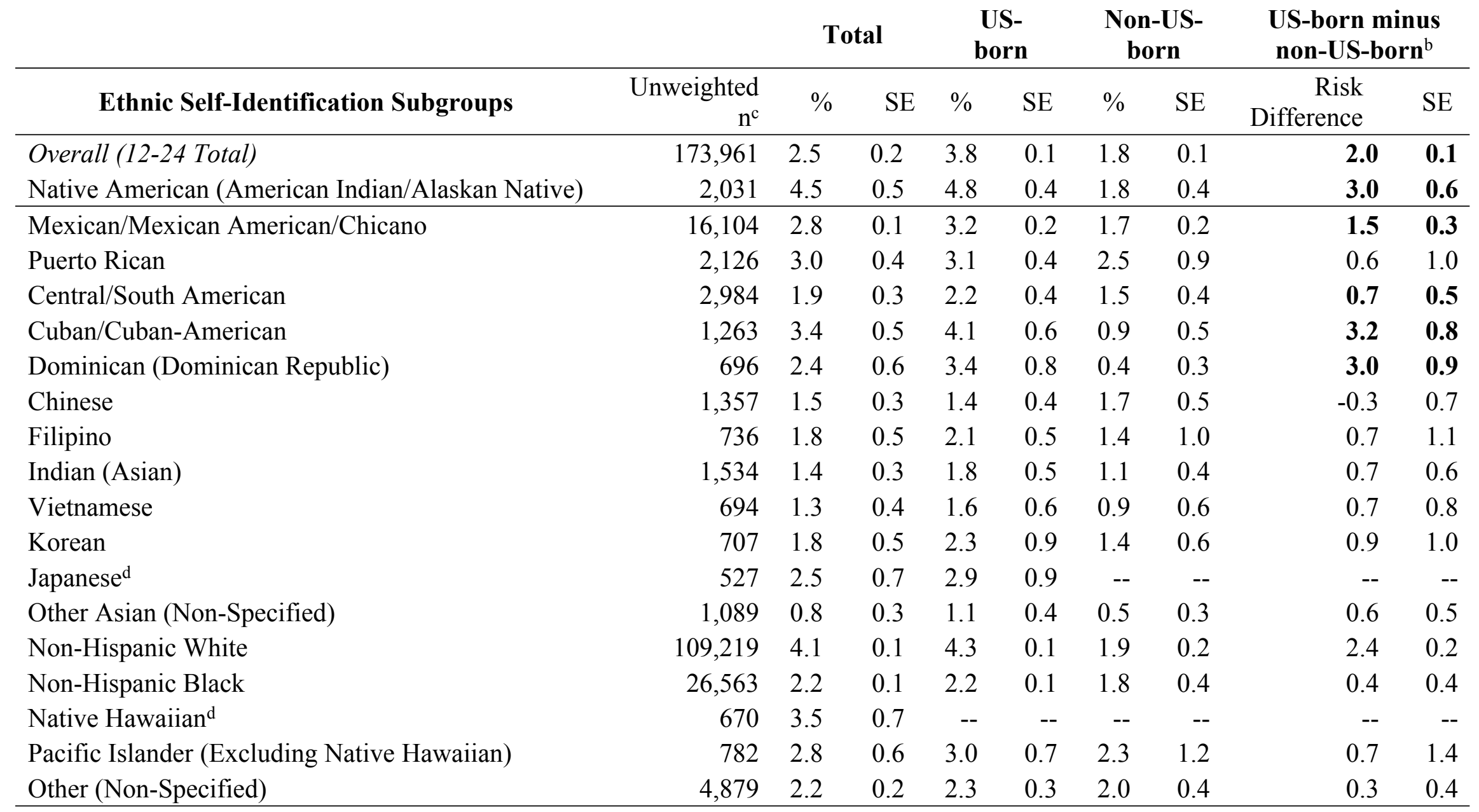


Figure 1

Estimated differences in the annual incidence rates and risk differences for extramedical prescription pain reliever use among 12-24-year-olds by ethnic selfidentification subgroups and birthplace.

Incidence estimates and RD are shown in two dimensions. The $\mathrm{x}$-axis arranges incidence estimates for US-born of each subgroup from smallest to largest. The $y$-axis presents estimated $\mathrm{RD}$ and $95 \% \mathrm{Cl}$.

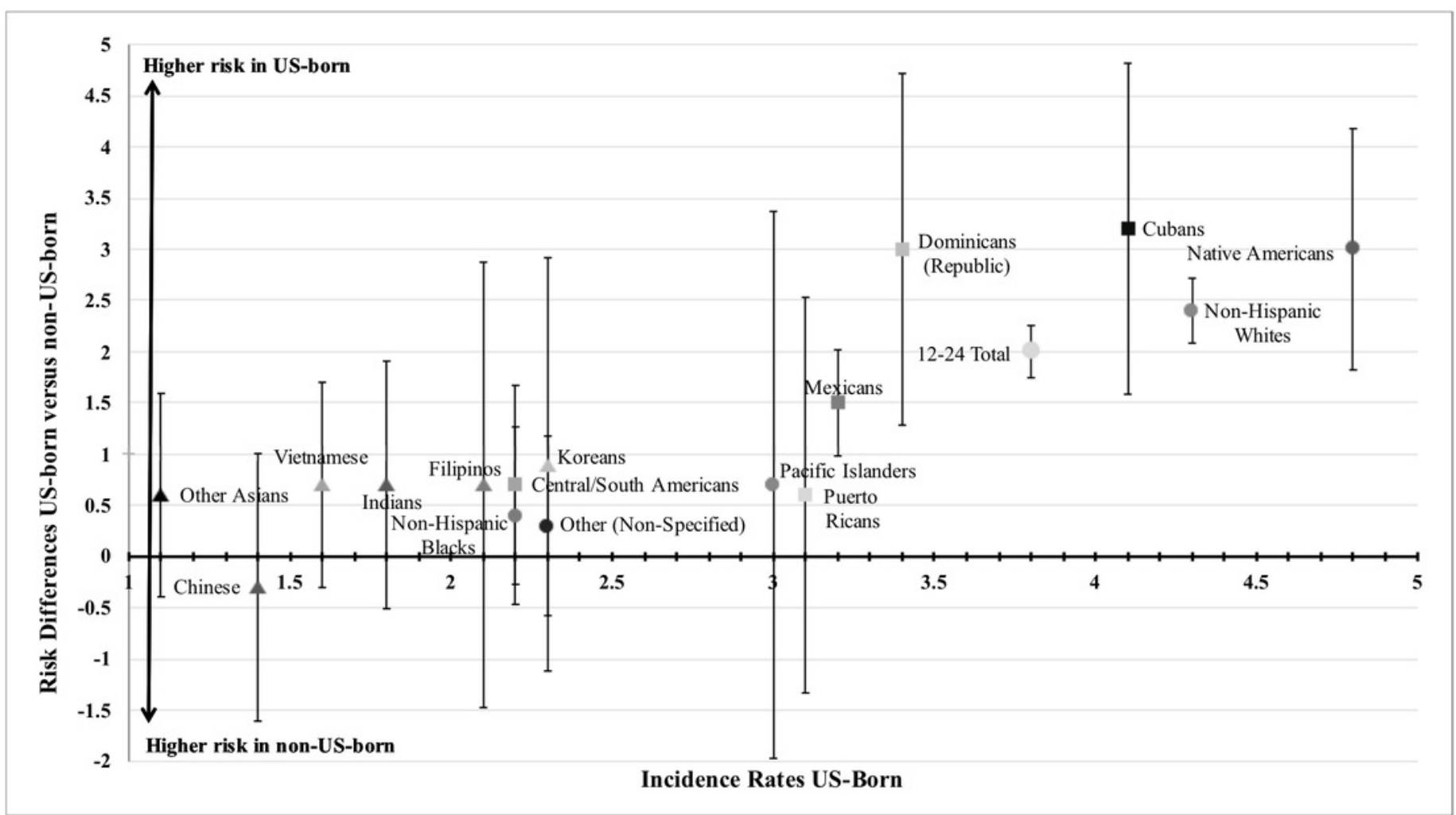

